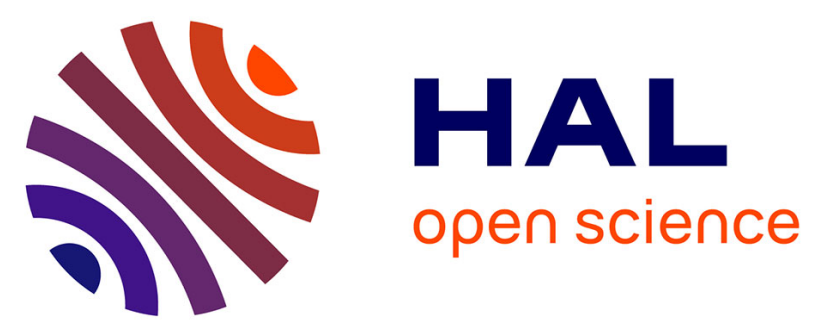

\title{
Conception simultanée de systèmes électriques hétérogènes par algorithmes évolutionnaires multicritères. Applications à l'optimisation de chaînes de traction pour véhicules électriques
}

Bruno Sareni, Jérémi Regnier, Xavier Roboam

\section{To cite this version:}

Bruno Sareni, Jérémi Regnier, Xavier Roboam. Conception simultanée de systèmes électriques hétérogènes par algorithmes évolutionnaires multicritères. Applications à l'optimisation de chaînes de traction pour véhicules électriques. Revue des Sciences et Technologies de l'Information - Série TSI : Technique et Science Informatiques, 2006, vol. 25, pp. 1103-1126. 10.3166/tsi.25.1103-1126 . hal-00847256

\section{HAL Id: hal-00847256 \\ https://hal.science/hal-00847256}

Submitted on 23 Jul 2013

HAL is a multi-disciplinary open access archive for the deposit and dissemination of scientific research documents, whether they are published or not. The documents may come from teaching and research institutions in France or abroad, or from public or private research centers.
L'archive ouverte pluridisciplinaire HAL, est destinée au dépôt et à la diffusion de documents scientifiques de niveau recherche, publiés ou non, émanant des établissements d'enseignement et de recherche français ou étrangers, des laboratoires publics ou privés. 


\title{
Conception simultanée de systèmes électriques hétérogènes par algorithmes évolutionnaires multicritères
}

\section{Application à l'optimisation de chaînes de traction pour véhicules électriques}

\author{
B. Sareni, J. Régnier, X. Roboam \\ Laboratoire d'électrotechnique et d'électronique industrielle \\ Unité mixte de recherche INPT-ENSEEIHT/CNRS \\ BP 7122, 2 rue Camichel, F-31071 Toulouse cedex 7 \\ \{sareni, regnier, roboam\}@leei.enseeiht.fr
}

\begin{abstract}
RÉSUMÉ. Dans cet article, des algorithmes évolutionnaires sont appliqués à l'optimisation multicritère d'une chaîne de traction pour véhicules électriques. Dans une première partie, la notion de conception simultanée de systèmes hétérogènes en génie électrique est développée et analysée. Les problèmes d'optimisation induits par cette approche sont assez complexes et les algorithmes évolutionnaires multicritères semblent bien adaptés pour fournir des solutions intéressantes au concepteur. Par la suite, un état de l'art de ces techniques est développé et un exemple d'application est donné. Il s'agit de concevoir des chaînes de traction pour véhicules électriques en fonction de missions spécifiques (cycle urbain ou routier). Les résultats obtenus soulignent l'intérêt de l'approche évolutionnaire multicritère pour la conception simultanée tant au niveau de l'optimisation que de l'analyse systémique de dispositifs complexes.
\end{abstract}

ABSTRACT. In this paper, evolutionary algorithms are applied to the multiobjective optimization of a traction system for electrical vehicles. First of all, the concept of integrated design of heterogeneous systems in electrical engineering is defined and analyzed. The optimization problems resulting from this approach are rather complex and evolutionary algorithms seem to be suitable to provide interesting solutions to the designer. Then, an overview of these techniques is presented and an application example is given. It consists in designing traction systems for electrical vehicle in relation to specific driving mission (urban or road driving cycles). The obtained results point out the interest of using multiobjective evolutionary algorithms in integrated design for optimization and complex system analysis.

MOTS-CLÉS : algorithmes évolutionnaires, optimisation multicritère, conception simultanée, approche système, véhicules électriques, génie électrique

KEYWORDS: evolutionary algorithms, multiobjective optimization, integrated design, system viewpoint, electrical vehicles, electrical engineering 


\section{Introduction}

La détermination de solutions industrielles innovantes pour les systèmes énergétiques complexes passe par l'amélioration des outils et des méthodes de conception. Il est notamment impératif de considérer les systèmes dans leur globalité pour garantir des performances optimales. En effet, l'optimisation «locale» des éléments d'un système pris indépendamment, n'assure aucunement l'optimalité de l'ensemble. Les couplages existant entre les divers éléments peuvent évidemment influer sur l'efficacité globale. De plus, il est préférable de mettre au même niveau dans le processus de conception les choix à faire en matière d'architecture, de dimensionnement et de gestion d'énergie, car ces trois facteurs sont fortement couplés aux performances.

C'est dans cette optique que nous pensons qu'il est nécessaire d'évoluer vers une conception simultanée qui vise, conjointement et de manière globale, à la détermination de l'architecture, du dimensionnement et de la gestion énergétique de l'ensemble des éléments d'un système. Ce type d'approche conduit inévitablement vers des problèmes d'optimisation complexes et spécifiques lorsque l'on recherche des configurations optimales du système. Les méthodes de résolution «exactes » sont trop limitées pour traiter ces problèmes, généralement à variables mixtes, contraints et multicritères. Par contre, les techniques stochastiques et en particulier les algorithmes évolutionnaires offrent des perspectives intéressantes.

Le travail présenté dans cet article illustre l'application d'algorithmes évolutionnaires multicritères à la résolution d'un problème typique de conception simultanée. Nous traitons le cas du dimensionnement d'une chaîne de traction pour véhicules électriques.

Dans une première partie, nous définissons plus précisément la notion de conception simultanée et détaillons les caractéristiques de cette approche. Nous dressons ensuite un bref état de l'art des algorithmes évolutionnaires multicritères et montrons comment ils peuvent être adaptés pour traiter ce type de problème. Enfin, nous donnons un exemple d'application à travers la conception de chaînes de traction pour véhicules électriques, destinés à remplir des cycles de circulation urbains ou routiers.

\section{Conception simultanée en génie électrique}

\subsection{Problématique de la conception simultanée des systèmes énergétiques}

La conception des systèmes d'énergie électrique représente aujourd'hui un véritable enjeu sociétal. Il s'agit notamment de répondre à une forte demande en matière de besoins énergétiques et de satisfaire à une performance toujours croissante des systèmes, tout en garantissant une gestion optimale de l'énergie. En effet, à l'inverse des dispositifs actuels souvent surdimensionnés par rapport à leur 
besoin, on cherche désormais à concevoir des systèmes « au plus juste » pour éviter tout gaspillage énergétique ou économique. Les difficultés rencontrées par le concepteur vis-à-vis de l'optimisation des dispositifs énergétiques sont liées à deux aspects principaux :

- Ces systèmes présentent un fort degré de complexité car eux-mêmes sont constitués d'un ensemble d'éléments (ou de sous-systèmes) dont l'architecture et le dimensionnement sont à déterminer pour atteindre des performances optimales.

- Ces systèmes sont fortement hétérogènes et multidisciplinaires. Les constantes de temps associées aux phénomènes physiques qui les caractérisent sont multiples et réparties. Ceci amène le concepteur à se poser la question du niveau de représentation pour chacun des éléments du système et du choix du type de modélisation (analytique, numérique de type algébro-différentiel ou éléments finis,...) en fonction d'un compromis lié à la précision des modèles et au coût en temps de calcul.

L'ensemble des difficultés relatives à la modélisation ne fait que complexifier la phase d'optimisation de ces systèmes. Classiquement, cette problématique est généralement simplifiée en adoptant une démarche séquentielle descendante, qui consiste d'abord à optimiser l'architecture du système, puis le dimensionnement des éléments, et enfin le choix de la stratégie de gestion de l'énergie. Néanmoins, en raison des couplages existant entre ces différents facteurs et de leur influence sur les performances du système, nous constatons bien qu'il est plus approprié d'adopter une approche d'optimisation globale. C'est bien là l'objectif de la conception simultanée qui, comme son nom l'indique, vise à intégrer de façon simultanée l'architecture, le dimensionnement et la gestion de l'énergie dans le système. Nous symbolisons les différences entre ces deux approches de conception à la Figure 1.

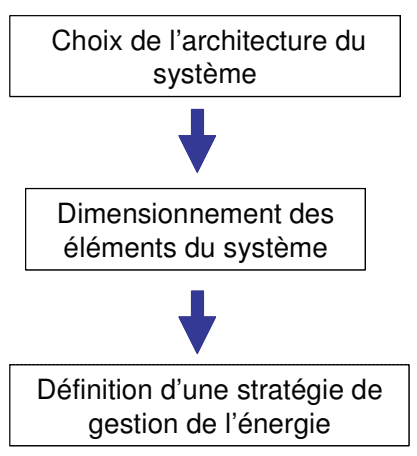

(a) approche séquentielle descendante

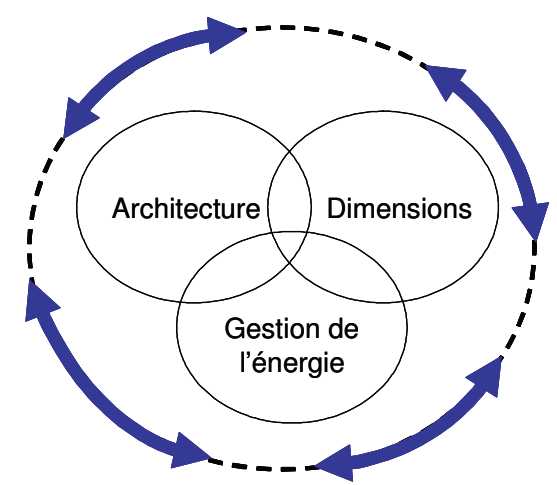

(b) conception simultanée

Figure 1. Approches de conception des systèmes énergétiques complexes 


\subsection{Exemple du véhicule électrique}

Le véhicule électrique "pur » est un exemple typique de système énergétique à caractère complexe et fortement hétérogène. Il est constitué d'un ensemble d'éléments comprenant principalement le châssis et la chaîne de traction électrique, elle-même composée d'une source d'énergie principale (généralement une batterie d'accumulateurs) et d'un convertisseur statique alimentant un moteur électrique. Ce dernier permet de transférer la puissance aux roues, souvent à travers un réducteur de vitesse et une transmission mécanique.

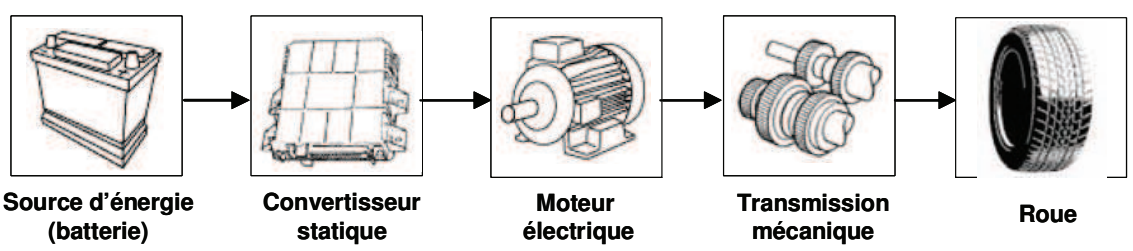

Figure 2. Synoptique d'une chaîne de traction pour véhicule électrique "pur »

Notons que l'architecture de la chaîne de traction électrique présentée au synoptique de la Figure 2 peut être plus complexe dans le cas de véhicules multisources ou hybrides (véhicules à sources d'énergie diverses: thermique, électrochimique, solaire,...) et de solutions multi-moteurs (un moteur par roue motrice). Notre choix s'est initialement et délibérément porté vers le véhicule électrique pur dont nous maîtrisons bien le comportement, ceci afin d'appréhender pleinement les mécanismes qui résultent du processus de conception simultanée par optimisation, selon les algorithmes et techniques employés.

\subsection{Conception simultanée par optimisation}

La problématique de la conception simultanée peut être traitée à l'aide de techniques d'optimisation. La formulation d'un cahier des charges associé à ce type d'approche globale conduit toutefois à des problèmes d'optimisation complexes et difficiles. Ces problèmes sont habituellement caractérisés par :

- un nombre important de variables de conception de nature discrète (paramètres combinatoires liés aux choix de structure et d'architecture, au type d'éléments ou de matériaux...) et de nature continue (paramètres de dimensionnement, variables énergétiques,...).

- de multiples contraintes intrinsèques au système global, à chaque sous-système ou liées à la compatibilité d'association entre éléments dans le système.

- diverses grandeurs à optimiser, typiquement des critères énergétiques (rendement, consommation, pertes,...), de dimensionnement (volume, masse, ...) ou économiques. 
Nous remarquons donc que la conception simultanée conduit à des problèmes d'optimisation, multicritères, à variables mixtes, sous contraintes. Aujourd'hui, les algorithmes évolutionnaires sont parmi les techniques les mieux appropriées à la résolution de tels problèmes. Dans la suite de cet article, nous dressons un bref état de l'art de l'optimisation multicritère par algorithmes évolutionnaires et soulignons notamment certaines particularités en vue de la conception simultanée.

\section{Optimisation multicritère par algorithmes évolutionnaires}

\subsection{Formulation d'un problème multicritère et optimalité au sens de Pareto}

L'optimisation multicritère (ou optimisation multiobjectif ${ }^{1}$,) vise à la minimisation simultanée d'un ensemble de $n$ critères $f$, fonction d'un nombre $m$ de paramètres (typiquement les variables de conception $\mathbf{X}$ aussi appelées variables de décision). Ces paramètres peuvent éventuellement être soumis à une série de contraintes d'inégalités $g$. Dans ce cas, le problème d'optimisation peut être formulé de la manière suivante :

$$
\begin{aligned}
& \text { Minimiser } f(\mathbf{X})=\left(f_{1}(\mathbf{X}), f_{2}(\mathbf{X}), \ldots, f_{n}(\mathbf{X})\right) \\
& \text { avec } g_{i}(\mathbf{X}) \leq 0 \text { pour } i=1 \ldots k
\end{aligned}
$$

Pour ce type de problèmes, les critères sont généralement conflictuels, de sorte que dans la plupart des cas, il est impossible de trouver un même point $\mathbf{X}$ qui minimise tous les critères simultanément. Par conséquent, le problème d'optimisation ne possède pas une solution unique mais un ensemble de solutions traduisant les meilleurs compromis possibles. Ces solutions sont qualifiées de solutions Pareto-optimales en référence au célèbre économiste italien (Pareto, 1896). Du point de vue mathématique, l'optimalité au sens de Pareto peut être exprimée en terme de dominance. Considérons par exemple, deux vecteurs solutions $\mathbf{X}$ et $\mathbf{Y}$; on dira que $\mathbf{X}$ domine $\mathbf{Y}$ si et seulement si :

$$
\forall i=1 \ldots n \quad f_{i}(\mathbf{X}) \leq f_{i}(\mathbf{Y}) \quad \text { et } \quad \exists j \in 1 \ldots n \Rightarrow f_{j}(\mathbf{X})<f_{j}(\mathbf{Y})
$$

Un vecteur qui n'est dominé par aucun élément d'un ensemble d'autres vecteurs est qualifié de non dominé vis-à-vis de cet ensemble. Les vecteurs qui sont non dominés sur l'ensemble de l'espace de recherche représentent les solutions Paretooptimales et constituent le front optimal de Pareto. Nous illustrons à la Figure 3, le concept de dominance au sens de Pareto dans le cas de deux critères $(n=2)$, en montrant la dominance d'une solution $\mathbf{X}$ quelconque vis-à-vis d'une solution $\mathbf{Y}$ donnée.

1.Nous utiliserons de façon équivalente critères et objectifs dans la suite de l'article. 


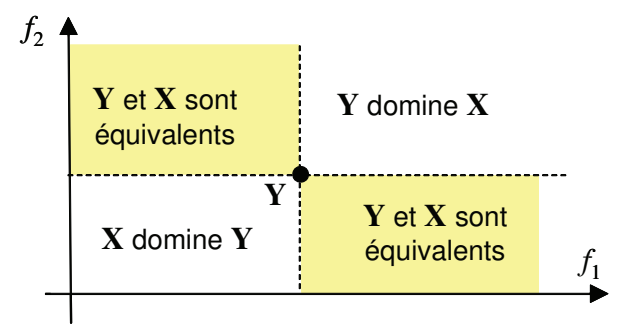

Figure 3. Illustration de la dominance au sens de Pareto - Minimisation de deux critères

\subsection{Méthodes de résolution des problèmes multicritères}

Les méthodes d'optimisation multicritère visent à fournir au concepteur une ou plusieurs solutions Pareto-optimales. Elles sont habituellement divisées en 3 grandes classes (Van Veldhuizen, 1999), (Colette et al., 2002) liées au processus de décision associé au choix de la solution finale.

- Les approches a priori regroupent l'ensemble des techniques pour lesquelles le choix du compromis à rechercher par optimisation est fait a priori par le concepteur. Ces méthodes incluent notamment la méthode de la contrainte $\mathcal{\varepsilon}$ (aussi appelée $\mathcal{\varepsilon}$ contrainte ou méthode des objectifs bornés) et l'ensemble des méthodes scalaires agrégatives (en particulier les méthodes de pondération des critères, les méthodes floues et la méthode du but à atteindre). Bien qu'elles aient été largement utilisées par le passé, ces techniques présentent un inconvénient majeur. En effet, dans le cas où les critères n'ont pas les mêmes dimensions physiques, elles fournissent au concepteur une unique solution dont la sensibilité est généralement importante vis-àvis des paramètres utilisés pour la mise à l'échelle et l'agrégation des critères (coefficients de pondération, facteurs de normalisation, valeurs cibles,...). Par ailleurs, il est souvent difficile pour le concepteur de fixer l'ensemble de ces paramètres a priori.

- Les approches progressives ou séquentielles procèdent par optimisations successives pour fournir une solution au problème. Le concepteur affine son choix de compromis à chaque optimisation.

- Les approches a posteriori ont pour caractéristique de fournir au concepteur un ensemble de compromis optimaux parmi lesquels il pourra extraire la solution de son problème. Elles regroupent essentiellement les métaheuristiques qui utilisent le concept de dominance au sens de Pareto et en particulier les algorithmes évolutionnaires qui feront l'objet de la section suivante. Ces techniques à base de population (ou qui exploitent la recherche en parallèle de plusieurs solutions Paretooptimales) ont l'avantage de ne nécessiter aucune mise à l'échelle des critères. Nous 
pouvons par ailleurs noter que les méthodes a priori peuvent être utilisées séquentiellement pour former des méthodes progressives ou a posteriori.

\subsection{Algorithmes évolutionnaires multicritères}

Les algorithmes évolutionnaires multicritères ont connu récemment un fort développement. En effet, ces méthodes sont particulièrement bien adaptées à la recherche de compromis optimaux en raison de leur capacité à explorer plusieurs solutions en parallèle.

\subsubsection{Les différentes approches évolutionnaires multicritères}

Plusieurs méthodes évolutionnaires multicritères ont été développées ces dernieres années. Nous citons notamment :

- les algorithmes basés sur une sélection d'objectifs alternée (Schaffer, 1985), (Kursawe, 1992).

- les algorithmes à approches scalaires agrégatives (Van Veldhuizen, 1999), (Hajela et al., 1992).

- les algorithmes à sélection contrôlée de type crowding (Weile et al., 1996), (Noble, 2002), (Teich et al, 1997).

- les algorithmes non-élitistes basés sur des approches de Pareto exploitant la méthode de partage de l'adaptation (fitness sharing) dans l'espace des objectifs (Fonseca et al., 1993), (Srinivas et al., 1994), (Horn et al.).

- les algorithmes élitistes qui utilisent des techniques de clustering (Zitzler, 1999), (Zitzler et al., 2001), (Corne et al., 2001), (Deb et al., 2000).

Parmi ces différentes méthodes, les algorithmes élitistes s'affirment aujourd'hui comme les plus efficaces. Ils utilisent une population externe appelée archive qui préserve les solutions non-dominées au cours des générations. L'archive ayant une taille limitée, il devient nécessaire d'éliminer certaines solutions Pareto-optimales lorsque leur nombre est supérieur à la taille limite. Ceci est réalisé à l'aide d'une procédure de clustering qui préserve les solutions les plus représentatives et garantit une répartition uniforme des solutions sur l'ensemble du front de Pareto. Dans la section suivante, nous détaillons plus particulièrement le NSGA-II que nous avons exploité pour l'optimisation de chaînes de traction d'un véhicule électrique.

\subsubsection{Le NSGA-II (Non-dominated Sorting Genetic Algorithm)}

Une version élitiste du NSGA, proposée par Deb (Deb et al., 2000), s'affirme aujourd'hui comme une référence solide en matière d'optimisation évolutionnaire multicritère. Comme pour le NSGA, les individus de la population sont classés par ordre de dominance selon le front auquel ils appartiennent. Puis, un opérateur $F_{\text {distance }}$ est utilisé pour estimer la densité de solutions autour d'un élément de la population conformément au pseudo-code suivant. 


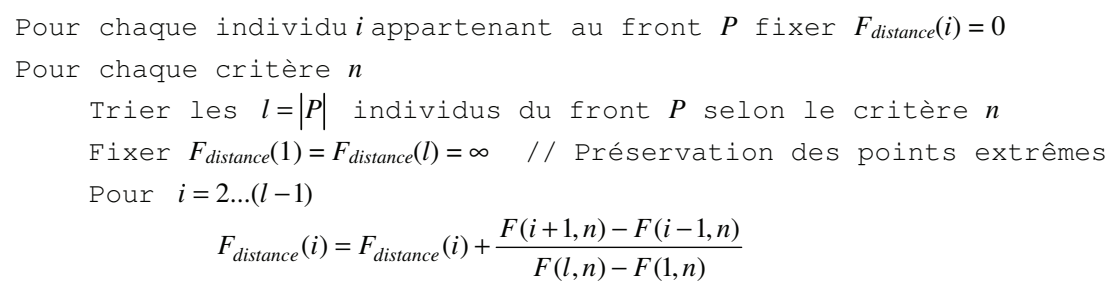

où $F(i, n)$ désigne la valeur du critère $n$ de l'individu $i$ appartenant au front $P$.

Notons que dans le calcul de $F_{\text {distance, }}$ nous avons choisi de normaliser chaque écart entre critères par l'écart maximal possible, pour réaliser une mise à l'échelle implicite. Ainsi, cet indicateur permet d'estimer la proximité d'un individu avec ses voisins, les individus isolés étant caractérisés par une $F_{\text {distance }}$ de forte valeur. Une des particularités du NSGA-II est qu'il possède une archive diversifiée de taille constante $N_{\text {arch }}$. Cette archive est remplie en insérant non seulement des individus non dominés de la population, mais aussi les individus des fronts successifs suivants, tant que leur nombre reste inférieur à la taille limite de l'archive. Lorsqu'il n'est pas possible d'insérer tous les individus d'un front dans l'archive (par manque de place), le choix entre les individus d'un même front se fait par troncation à partir de la $F_{\text {distance }}$ (les individus du front de plus forte $F_{\text {distance }}$ sont conservés en priorité). De cette manière, on préserve d'abord les solutions extrêmes puis celles qui sont les plus isolées. La sélection des parents géniteurs dans le NSGA-II est effectuée à partir de l'archive diversifiée en réalisant des tournois basés sur le front auquel appartiennent les individus. Lorsque ceux-ci font partie d'un même front, le choix se fait aussi relativement à la plus forte $F_{\text {distance }}$. Enfin, l'évolution de la population est régie par des mécanismes standards de croisement et de mutation. Nous utiliserons pour notre part l'opérateur de croisement auto-adaptatif décrit dans (Sareni et al., 2004). Cet opérateur permet notamment d'améliorer la robustesse du NSGA-II.

\subsection{Adaptation des algorithmes pour la conception simultanée}

Les particularités de la conception simultanée imposent certaines adaptations visà-vis de l'implantation des algorithmes évolutionnaires. Nous avons notamment signalé précédemment la nécessité de normaliser le critère de distance utilisé pour estimer la densité de solutions autour d'un individu. D'autres spécificités liées à la problématique de la conception simultanée sont à considérer, notamment la prise en compte des contraintes de conception et des aspects combinatoires.

\subsubsection{Prise en compte des contraintes de conception}

Contrairement aux problèmes d'optimisation mathématiques de test, il est généralement impossible d'évaluer les contraintes de conception en parallèle. Les modèles de dimensionnement imposent normalement d'appliquer certaines contraintes de façon séquentielle. En effet, on comprendra aisément qu'on ne peut pas tester des contraintes de bon fonctionnement du système global si un des éléments est lui-même non réalisable en raison d'autres contraintes «locales » non 
vérifiées. Par conséquent, on peut établir, pour tout problème, un graphe de contraintes lié aux modèles de dimensionnement et de simulation qui caractérise les couplages et les enchaînements entre contraintes (voir Figure 4). Ce graphe peut être décomposé en niveaux pour faciliter son analyse. Seules les contraintes appartenant à un même niveau peuvent être évaluées en parallèle. En outre, on notera que nous avons aussi représenté, dans le graphe de la Figure 4 , les critères de conception $\left(f_{1}\right.$ et $f_{2}$ ), eux-mêmes soumis aux règles précédentes. Ainsi, dans l'exemple donné, ces critères ne seront calculables que si les contraintes $g_{1}$ à $g_{6}$ sont vérifiées, la dernière contrainte $g_{7}$ pouvant être estimée en parallèle. Plus le nombre de niveaux dans le graphe est élevé, plus le problème d'optimisation est difficile. Par ailleurs, les contraintes peuvent être classées en deux groupes selon que leur détermination se fait avant ou après la simulation du système (coût principal en temps CPU). Les contraintes pré-simulation seront principalement des contraintes « locales », relatives aux éléments du système ou à leur association, tandis que les contraintes postsimulation caractériseront plutôt des contraintes «globales », s'appliquant à l'ensemble du système.

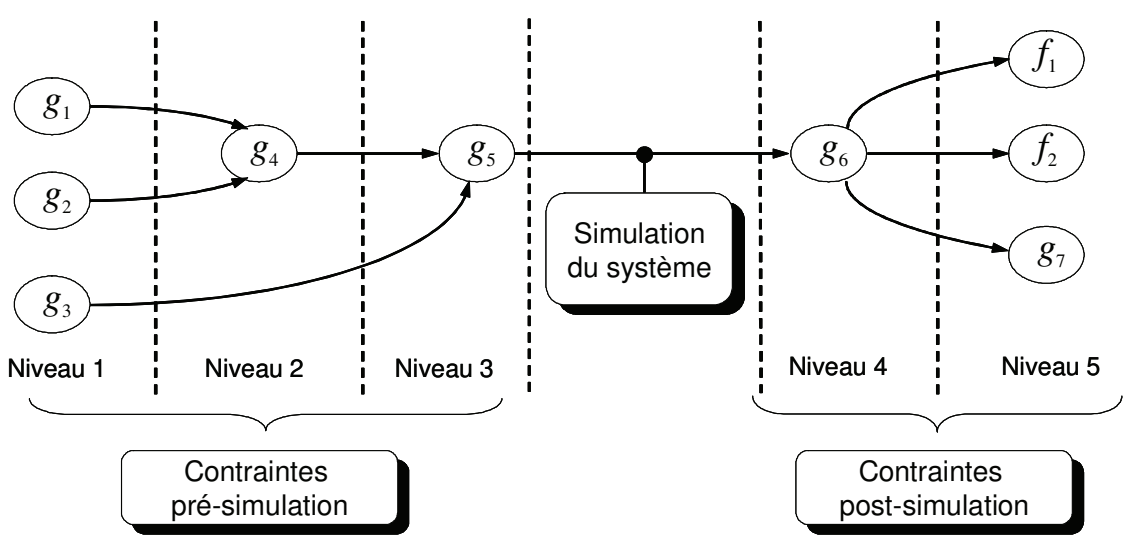

Figure 4. Exemple de graphe de contraintes en conception simultanée

La détermination séquentielle des contraintes complexifie largement le problème d'optimisation. Les contraintes violées constituent des « barrières » à l'algorithme de résolution qui se trouve, en outre, «aveugle » vis-à-vis des contraintes de niveaux supérieurs non calculables. Lorsqu'une contrainte n'est pas calculable (car dépendant d'une autre contrainte en amont non vérifiée), nous recommandons d'imposer pour celle-ci (ainsi que pour celles des niveaux supérieurs) une pénalisation radicale (death penalty) à savoir $g(\mathbf{X})=+\infty$. Pour prendre en compte les contraintes de conception dans l'algorithme évolutionnaire, nous avons choisi d'étendre les règles de dominance de la manière suivante : 
- si deux individus $\mathrm{A}$ et $\mathrm{B}$ sont non réalisables, la dominance de $\mathrm{B}$ vis-à-vis de $\mathrm{A}$ (ou celle de A vis-à-vis de B) est établie dans l'espace des contraintes.

- si deux individus A et B sont réalisables, la dominance de B vis-à-vis de A (ou celle de A vis-à-vis de B) est établie dans l'espace des critères

- tout individu non réalisable sera considéré dominé vis-à-vis d'un individu réalisable.

Ces règles permettent de conjuguer la minimisation des contraintes et la minimisation des critères en utilisant l'approche de Pareto dans les deux espaces. On notera dans le cas du NSGA-II que pour les individus non réalisables appartenant à un front donné dans l'espace des contraintes, le calcul de la $F_{\text {distance }}$ se fera relativement aux contraintes. De cette manière, l'opérateur de nichage permettra de garantir la diversité des solutions dans les deux espaces et limitera ainsi la convergence prématurée de l'algorithme.

\subsubsection{Prise en compte des aspects combinatoires}

Les aspects combinatoire et continu de la conception simultanée imposent d'avoir recours à un codage adapté permettant l'évolution par recombinaison et/ou mutation des variables discrètes et continues. La programmation génétique (Koza, 1992) et le codage par graphe peuvent être conjugués aux opérateurs évolutionnaires classiques dédiés à l'optimisation continue (Michalewicz, 1996). Dans le cas de problèmes fortement orientés vers le dimensionnement, où l'aspect architecture intervient faiblement et où les variables discrètes sont peu nombreuses, on peut toutefois simplifier l'implantation en gardant un algorithme standard à codage réel mais en décodant les variables discrètes à l'aide de la fonction « partie entière ».

\section{Application des algorithmes évolutionnaires à la conception de chaînes de traction pour véhicules électriques}

Nous présentons, dans cette partie, l'application d'algorithmes évolutionnaires multicritères à la conception d'une chaîne de traction pour véhicules électriques. De nombreuses recherches sur cette thématique sont en cours. Actuellement, les solutions les plus avancées et les plus viables économiquement sont des structures hybrides, qui mêlent une motorisation thermique, un alternateur ainsi qu'une machine électrique associée à une batterie. Les architectures «tout électrique » souffrent de problèmes liés au stockage de l'énergie embarquée. Les performances des accumulateurs en terme d'énergie massique sont encore bien faibles par rapport aux carburants pétroliers (Multon, 2001). La conception globale et optimisée de ces dispositifs peut permettre d'aboutir à des solutions innovantes. Nous avons retenu comme système d'étude un véhicule électrique «pur» dont la chaîne de traction électromécanique est similaire à celle de la Figure 2. Celle-ci est composée d'une batterie d'accumulateurs ions-Lithium, d'un convertisseur statique (onduleur de tension triphasé) permettant l'alimentation d'un moteur électrique synchrone 
éventuellement associé à un réducteur mécanique de rapport fixe. Nous préciserons quelques particularités du modèle, notamment les différentes variables de conception, ainsi que certaines données technologiques relatives au cahier des charges et au paramétrage du système, en vue de son optimisation. Deux critères devront être minimisés, à savoir les pertes totales dans le véhicule ainsi que la masse embarquée (masse globale de la chaîne de traction hors châssis et passagers).

\subsection{Modèle global du véhicule électrique}

Nous ne donnerons pas tous les détails des modèles des divers éléments du véhicule en raison de leur complexité. Ceux-ci relèvent d'ailleurs plus spécifiquement du domaine du génie électrique et ont déjà fait l'objet de publications antérieures (Régnier et al., 2003), (Régnier, 2003).

\subsubsection{Modèle de la batterie d'accumulateurs}

La batterie d'accumulateurs est constituée de $n_{p}$ branches en parallèle de $n_{s}$ cellules en série. Chaque cellule de la batterie peut être représentée par une résistance interne $R_{0}$, en série avec une fem $E_{0}$. Cette dernière est fonction de l'état de charge $E D C$ de la cellule et évolue au cours de la mission de circulation du véhicule. Pour des raisons technologiques, elle doit impérativement rester comprise entre deux valeurs fixées par le constructeur $\left(E_{0}^{\min } \leq E_{0} \leq E_{0}^{\max }\right)$. L'évolution de l'état de charge d'une cellule est liée au courant $I_{c e l}$ fourni (ou récupéré lors des phases de freinage) par la cellule et à sa capacité énergétique $C_{3}$ (capacité définie pour une décharge en 3 heures à courant constant $I_{3}=\mathrm{C}_{3} / 3$ ). L'état de charge des cellules est considéré maximal au début de la mission $\left(E D C=1\right.$ et $\left.E_{0}=E_{0}^{\max }\right)$. Trois types de cellules ions-lithium du constructeur SAFT sont utilisés pour l'optimisation des performances du véhicule. Leurs caractéristiques sont données au Tableau 1. Les pertes dans la batterie $P_{b a t}$ sont déduites des valeurs de résistances internes des cellules et du courant qui les parcourt. La masse de la batterie $M_{b a t}$ est calculée à partir de la masse d'une cellule et du nombre total de cellules.

\begin{tabular}{cccccc}
\hline $\begin{array}{c}\text { Type de } \\
\text { cellule }\end{array}$ & Capacité & Résistance & \multirow{2}{*}{ Masse } & \multicolumn{2}{c}{ f.e.m. potentielle } \\
$\mathrm{C}_{3}$ & Interne $R_{0}$ & & $E_{0}^{\min }$ & $E_{0}^{\max }$ \\
\hline 1 & $39 \mathrm{~A} . \mathrm{h}$ & $7 \mathrm{~m} \Omega$ & $1.05 \mathrm{~kg}$ & $2.7 \mathrm{~V}$ & $4 \mathrm{~V}$ \\
\hline 2 & $25 \mathrm{~A} . \mathrm{h}$ & $16.75 \mathrm{~m} \Omega$ & $0.75 \mathrm{~kg}$ & $2.7 \mathrm{~V}$ & $4 \mathrm{~V}$ \\
\hline 3 & $16 \mathrm{~A} . \mathrm{h}$ & $23 \mathrm{~m} \Omega$ & $0.68 \mathrm{~kg}$ & $2.7 \mathrm{~V}$ & $4 \mathrm{~V}$ \\
\hline
\end{tabular}

Tableau 1. Caractéristiques des cellules élémentaires de la batterie 


\subsubsection{Modèle de l'onduleur de tension}

L'alimentation du moteur électrique est assurée par un onduleur de tension piloté en courant fonctionnant à la fréquence de découpage $F_{d e c}$. Il est composé de six semiconducteurs de type IGBT, équipés de diodes montées en parallèle et destinées à assurer la réversibilité en courant des interrupteurs. Les pertes dans l'onduleur se répartissent en pertes par conduction $P_{\text {cond }}$ et pertes par commutation $P_{\text {com }}$. Les pertes par conduction sont fonction du facteur de modulation, du courant maximal circulant dans la machine et des caractéristiques des diodes et IGBT (résistances internes, tensions à l'état passant,...). Les pertes par commutation sont évaluées à partir de la fréquence de découpage $F_{d e c}$ et des notices techniques du constructeur, par extrapolation des courbes d'énergie à l'amorçage et au blocage des IGBT. Un modèle thermique du composant permet de déduire la température au niveau des modules IGBT-diode, ainsi que la résistance thermique du radiateur pour garantir un fonctionnement aux conditions de température spécifiées (la température des modules ne devant pas excéder $100^{\circ} \mathrm{C}$ ). La connaissance de cette résistance thermique fixe par la suite les dimensions du radiateur (en particulier sa longueur) et sa masse $M_{r a d}$, représentative de la masse de l'onduleur. Pour l'optimisation du véhicule électrique, 3 types de modules IGBT du constructeur IXYS (référencés MII200-12A4, MII300-12A4, MII400-12E7) seront considérés.

\subsubsection{Modèle du moteur électrique synchrone}

Le modèle du moteur électrique synchrone est donné dans (Slemon et al., 92). Il dépend principalement de paramètres géométriques (le nombre $p$ de paires de pôle, le nombre d'encoches par pôle et par phase $N_{e p p}$, le rapport rayon sur longueur $R_{r l}$ ) et électromécaniques (la densité de courant $J_{s}$, le couple de dimensionnement de base $C_{\text {dim }}$, la vitesse de dimensionnement de base $\Omega_{\text {dim }}$ ). Le moteur est piloté avec une stratégie à couple maximal par ampère pour atteindre les références en couple et en vitesse requises lors de la mission du véhicule. Les pertes dans le moteur sont constituées des pertes électriques par effet Joule $P_{j}$ et des pertes magnétiques dans le fer $P_{f}$ (Hoang, 1995). Un modèle thermique permet par ailleurs d'avoir la température des différents composants du moteur (bobinage, isolant, couronne statorique) en fonction de leurs caractéristiques thermiques (conductivité et capacité thermique des matériaux) et des conditions extérieures (température ambiante). Ce modèle est couplé aux caractéristiques électromagnétiques du moteur au travers des pertes Joule et des pertes fer. La masse totale de la machine est obtenue à partir de ses paramètres géométriques et de la masse volumique des divers matériaux qui le constituent (fer, aimant, cuivre).

\subsubsection{Modèle du réducteur mécanique de vitesse}

Un réducteur à engrenage cylindrique droit (Le Borzec, 1999) caractérise la chaîne mécanique du moteur à la roue. Un modèle simplifié permet de déduire à partir de paramètres de dimensionnement (à savoir le couple de dimensionnement $C_{r e d}$ et le rapport de réduction $N$ ) la masse du réducteur $M_{\text {red }}$ et les pertes $P_{\text {red }}$ qui lui sont associées. 


\subsubsection{Modèle mécanique du véhicule}

Afin de pouvoir simuler le parcours du véhicule, il est nécessaire de connaître les couples et les vitesses imposés au moteur de traction. Ces grandeurs dépendent en particulier de la mission (profils de vitesse), des caractéristiques de la route (profils de pente), des conditions extérieures (vitesse du vent, adhérence au sol,...), et de certaines données concernant le véhicule (voir Tableau 2). Un modèle mécanique (Gillespie, 1992) traduisant l'ensemble des efforts appliqués au véhicule (poids, force de résistance au roulement, force de résistance aérodynamique, dynamique d'accélération) permet alors de fournir les références de couple et de vitesse au moteur de traction.

\begin{tabular}{ll}
\hline Masse à vide du châssis & $M=800 \mathrm{~kg}$ \\
\hline Section frontale & $S=2 \mathrm{~m}^{2}$ \\
\hline Coefficient de pénétration dans l'air & $C_{\mathrm{x}}=0.4$ \\
\hline Rayon de roue & $R_{\text {roue }}=0.33 \mathrm{~m}$ \\
\hline
\end{tabular}

Tableau 2. Données relatives au modèle mécanique du véhicule

\subsubsection{Missions de circulation}

Les missions choisies, établies par l'INRETS (Institut National de Recherche sur les Transports et la Sécurité), proviennent de données statistiques portant sur un ensemble de cycles de circulation réels (Trigui, 1996). Les profils de pente sont considérés plats.

- La mission urbaine décrite par la Figure 5 est caractérisée par des vitesses faibles (autour de $40 \mathrm{~km} / \mathrm{h}$ ) avec une pointe de vitesse à environ $90 \mathrm{~km} / \mathrm{h}$. Le pas d'échantillonnage de la mission est de 1 seconde. Nous illustrons aussi l'allure du couple aux roues imposé par la mission pour une masse totale du véhicule de $1000 \mathrm{~kg}$ (soit $200 \mathrm{~kg}$ de masse embarquée). La durée de cette mission type est de 1374 secondes (soit environ 23 minutes), durant lesquelles le véhicule parcourt $12.2 \mathrm{~km}$. Pour assurer une autonomie de $200 \mathrm{~km}$, le profil élémentaire sera répété 16 fois.

- La mission routière présente des vitesses plus élevées pouvant atteindre jusqu'à $120 \mathrm{~km} / \mathrm{h}$. De la même manière que pour la mission urbaine, nous donnons, à la Figure 6, l'allure du couple aux roues pour une masse totale du véhicule de $1000 \mathrm{~kg}$. La durée de cette mission est de 734 secondes (soit environ 12 minutes) durant lesquelles le véhicule parcourt $15.4 \mathrm{~km}$. Il faut dans ce cas répéter 13 fois le profil pour garantir la même autonomie que précédemment. 

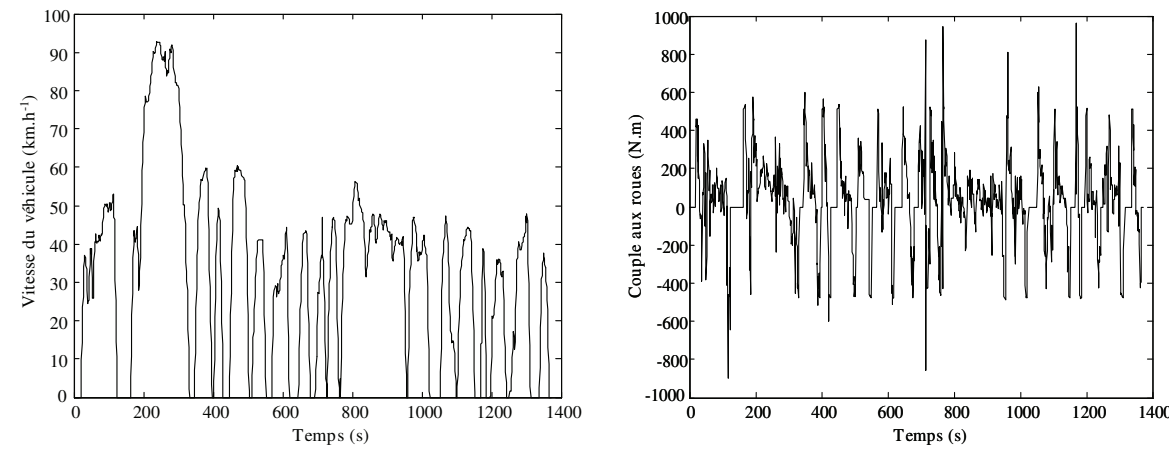

Figure 5. Caractéristiques de la mission urbaine
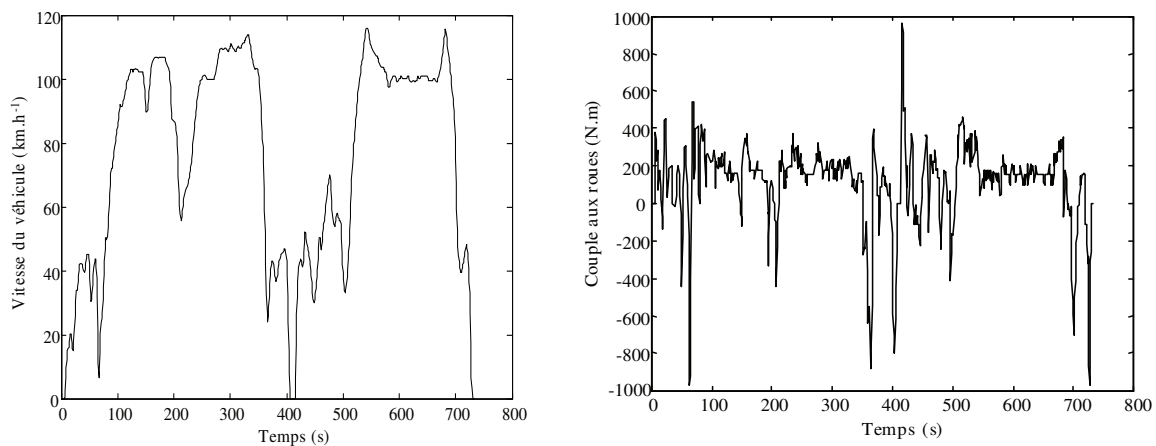

Figure 6. Caractéristiques de la mission routière

\subsection{Variables de conception du problème}

Les variables de conception du problème, ainsi que leurs domaines de variation, sont résumés dans le Tableau 3. Nous constatons par ailleurs que sept variables associées aux paramètres de dimensionnement sont de nature continue alors que six variables liées à la structure et à l'architecture du système sont discrètes.

\subsection{Contraintes de conception}

Douze contraintes de conception doivent être vérifiées pour assurer la « viabilité » du véhicule électrique et permettre à ce dernier de remplir sa mission. A partir des modèles de dimensionnement et de simulation, nous pouvons établir un graphe de contraintes décomposable en trois niveaux. 


\begin{tabular}{|c|c|c|}
\hline Variable de conception & Nature & Domaine de variation \\
\hline \multicolumn{3}{|c|}{ Batterie } \\
\hline Nombre de cellules en série & discrète & $n_{s} \in\{1, . .200\}$ \\
\hline Nombres de branches en parallèle & discrète & $n_{p} \in\{1, \ldots, 200\}$ \\
\hline Type de cellules & discrète & Type_cellule $\in\{1,2,3\}$ \\
\hline \multicolumn{3}{|c|}{ Onduleur } \\
\hline Fréquence de découpage & continue & $F_{d e c} \in[0.001,100][\mathrm{kHz}]$ \\
\hline Type de modules IGBT & discrète & Type_inter $\in\{1,2,3\}$ \\
\hline \multicolumn{3}{|c|}{ Machine synchrone à aimants permanents } \\
\hline Rapport rayon sur longueur & continue & $R_{r} \in[0.1,10]$ \\
\hline Nombre de paires de pôles & discrète & $p \in\{1, \ldots, 10\}$ \\
\hline Nombre d'encoches / pôle / phase & discrète & $N_{e p p} \in\{1,2,3\}$ \\
\hline Densité de courant & continue & $J_{s} \in[1,10] \quad\left[\mathrm{A} / \mathrm{mm}^{2}\right]$ \\
\hline Couple de dimensionnement & continue & $C_{d i m} \in[1,2000] \quad[\mathrm{N} . \mathrm{m}]$ \\
\hline Vitesse de dimensionnement & continue & $\Omega_{\text {dim }} \in[1,10000] \quad[\mathrm{rad} / \mathrm{s}]$ \\
\hline \multicolumn{3}{|c|}{ Réducteur mécanique de vitesse } \\
\hline Rapport de réduction & continue & $N \in[1,10]$ \\
\hline Couple de dimensionnement & continue & $C_{r e d} \in[1,2000] \quad[$ N.m $]$ \\
\hline
\end{tabular}

Tableau 3. Variables de conception du véhicule électrique

- Le niveau 1 est constitué de deux contraintes $g_{1}$ et $g_{2}$ associées au dimensionnement de la machine électrique. Elles concernent le nombre de conducteurs par encoche (déterminé à partir du nombre d'encoches par pôle et par phase et des autres caractéristiques géométriques de la machine). Ce nombre doit évidemment être supérieur à 1 et est limité par la section d'encoche en fonction de la section minimale des conducteurs (celle-ci est fixée à $0.5 \mathrm{~mm}^{2}$ ).

- Les contraintes du niveau suivant $g_{3}$ et $g_{4}$ s'assurent du bon fonctionnement de la machine en régime permanent à son point de dimensionnement $\left(C_{d i m}\right.$ et $\left.\Omega_{d i m}\right)$. En particulier, la température du bobinage en ce point ne doit pas dépasser $100^{\circ} \mathrm{C}$ et l'induction dans les aimants doit être limitée à $-0.2 \mathrm{~T}$ pour éviter leur désaimantation.

- Enfin, les contraintes du niveau 3 vérifient que le système est capable de remplir sa mission. Elles sont calculées pour chaque point de la mission au cours de la simulation du véhicule sur son parcours (urbain ou routier). Comme précédemment, nous devons nous assurer que la machine électrique satisfait la contrainte thermique et la contrainte de désaimantation $\left(g_{5}\right.$ et $\left.g_{6}\right)$. Elle doit en outre être capable d'atteindre les couples et vitesses imposés par la mission $\left(g_{7}\right)$. Deux contraintes supplémentaires $\left(g_{8}\right.$ et $\left.g_{9}\right)$ sont relatives à l'onduleur. La première garantit que les composants semiconducteurs ne sont pas soumis à des températures excédant $100^{\circ} \mathrm{C}$. La seconde limite la fréquence de découpage vis-à-vis de la fréquence maximale du fondamental dans la machine synchrone afin de réduire la distorsion harmonique. 
Deux autres contraintes $\left(g_{10}\right.$ et $\left.g_{11}\right)$ sont associées à la batterie : l'une concerne l'état de charge minimal à assurer lors du franchissement du profil, l'autre garantit de fournir suffisamment de puissance à la machine (compte tenu des pertes dans les résistances internes) pour qu'elle opère aux points de fonctionnement imposés par la mission. Enfin, la dernière contrainte $\left(g_{12}\right)$ liée au réducteur vise à vérifier que ce dernier ne subit pas des couples supérieurs à son couple de dimensionnement. Nous concluons cette partie en représentant à la Figure 7 le synoptique du modèle global du véhicule électrique en vue de son optimisation. Nous précisons notamment l'ensemble des variables, contraintes et critères de conception.

\subsection{Optimisation de véhicules électriques}

L'optimisation de véhicules électriques est réalisée avec le NSGA-II pour les deux missions de circulation (urbaine et routière) présentées précédemment. Les tailles de population et d'archive sont fixées à 100. Les opérateurs de mutation et de recombinaison sont identiques à ceux utilisés dans (Sareni et al., 2004) avec une probabilité de croisement de 1 , un taux de mutation des variables de conception de $1 / m$ ( $m$ désignant le nombre total de variables de conception du problème) et une probabilité de mutation de $5 \%$ pour le gène de croisement (paramètre d'autoadaptation entre les différents opérateurs de croisement). Le nombre de générations est égal à 1500. Dix exécutions distinctes sont réalisées pour s'assurer de la reproductibilité des résultats. On notera que le temps CPU nécessaire à l'évaluation des performances d'un individu réalisable ${ }^{2}$ sur un PC équipé d'un processeur $1 \mathrm{GHz}$ est d'environ 0.25 secondes. En conséquence, selon le nombre d'individus réalisables évalués au cours de l'optimisation, le temps CPU des dix exécutions du NSGA-II, compte tenu du paramétrage précédent (100 individus - 1500 générations ${ }^{3}$ ), peut varier de 1 à 4 jours ! Les fronts optimaux de Pareto donnés par le NSGA-II pour les deux missions de circulation considérées sont représentés à la Figure 8. Le domaine de variation des variables de conception correspondantes est donné dans le Tableau 4. Nous avons illustré par ailleurs, sur la Figure 8, la mise en situation des véhicules dimensionnés pour la mission routière lorsqu'ils effectuent la mission urbaine. Les solutions de la mission urbaine étant dimensionnées au plus juste, elles sont incapables de remplir les conditions routières plus contraignantes en puissance. Les résultats obtenus à la suite de l'optimisation montrent notamment l'importance de la prise en compte de la mission de circulation dans la détermination de performances optimales (ici définies en terme de masse et de pertes) pour le véhicule électrique.

2.Temps nécessaire au dimensionnement du véhicule, à l'évaluation des contraintes présimulation, à la simulation du véhicule sur son parcours, à l'évaluation des contraintes postsimulation et au calcul des critères de conception

3.On notera qu'avec ce paramétrage, l'algorithme a convergé vers le même front optimal (celui présenté à la figure 8) lors des dix exécutions indépendantes. Des essais, effectués avec un nombre de générations réduit (typiquement 500), n’ont pas permis à l'algorithme de converger de façon satisfaisante (Régnier, 2003). 


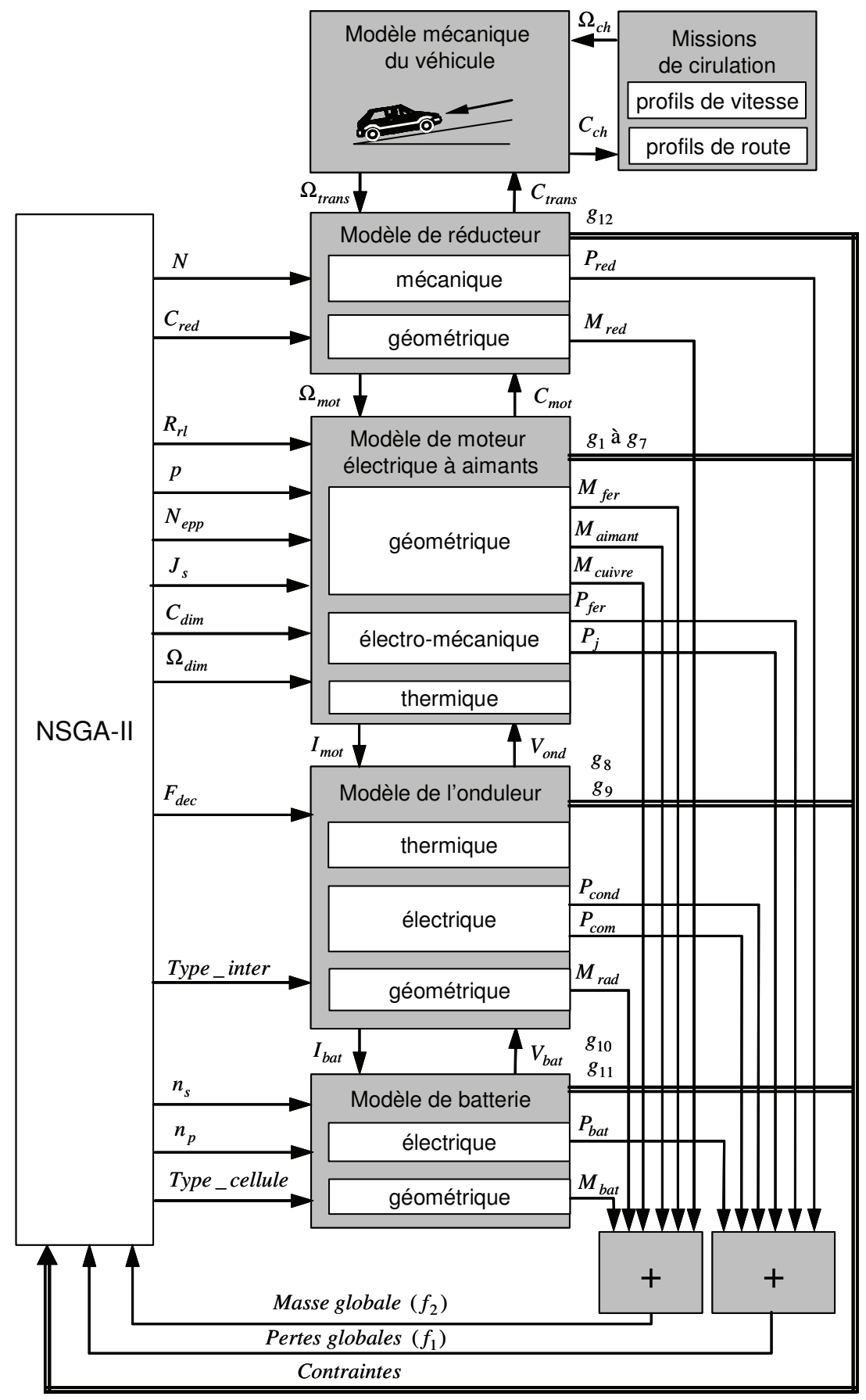

Figure 7. Modèle global du véhicule électrique en vue de son optimisation 


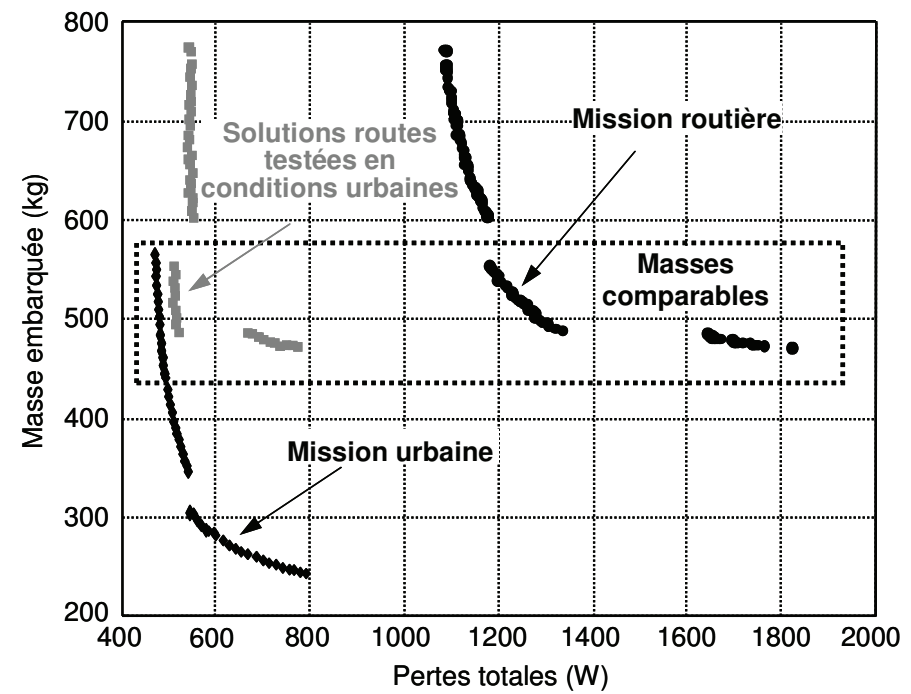

Figure 8. Solutions Pareto-optimales de véhicules électriques pour les missions de circulation urbaine et routière.

\begin{tabular}{ccccc}
\hline \multirow{2}{*}{ Variable de conception } & \multicolumn{2}{c}{ Mission urbaine } & \multicolumn{2}{c}{ Mission routière } \\
\cline { 2 - 5 } & $\min$ & $\max$ & $\min$ & $\max$ \\
\hline Nombre de cellules en série $n_{s}$ & 118 & 200 & 97 & 200 \\
\hline Nombres de branches en parallèle $n_{p}$ & 1 & 2 & 2 & 3 \\
\hline Type de cellules & \multicolumn{2}{c}{1 (39 A.h) } & \multicolumn{2}{c}{1 (39 A.h) } \\
\hline Fréquence de découpage $F_{\text {dec }}$ & 1195 & 2004 & 1273 & 1741 \\
\hline Type de modules IGBT & MII400-12E7 & MII300-12A4 \\
\hline Rapport rayon sur longueur $R_{r l}$ & 0.98 & 1.91 & 0.58 & 1.12 \\
\hline Nombre de paires de pôles $p$ & 5 & 7 & 4 & 4 \\
\hline Nombre d'encoches / pôle / phase $N_{e p p}$ & 1 & 1 & 1 & 1 \\
\hline Densité de courant $J_{s}$ & 1.31 & 2.15 & 1 & 1.075 \\
\hline Couple de dimensionnement $C_{\text {dim }}$ & 218 & 387 & 172 & 196 \\
\hline Vitesse de dimensionnement $\Omega_{\text {dim }}$ & 378 & 644 & 356 & 412 \\
\hline Rapport de réduction $N$ & 1 & 1 & 1 & 1 \\
\hline Couple de dimensionnement $C_{r e d}$ & pas de réducteur (transmission directe) \\
\hline
\end{tabular}

Tableau 4. Domaines de variation des variables de conception pour les solutions optimales 


\subsection{Capitalisation des résultats de l'optimisation multicritère pour l'analyse du système}

La conception simultanée à l'aide d'algorithmes évolutionnaires multicritères conduit donc à l'optimisation des performances globales du système par la détermination de plusieurs configurations Pareto-optimales. La connaissance de ces solutions permet en outre de déduire a posteriori des informations utiles concernant le système, particulièrement difficiles à établir avant la phase d'optimisation. Elles permettront au concepteur de l'aider dans la compréhension des relations entre variables, contraintes et critères de conception, de manière à faciliter l'analyse des couplages et de la sensibilité paramétrique vis-à-vis des performances. Nous illustrons brièvement ces points dans le cas des véhicules électriques conçus pour répondre à une mission de circulation urbaine.

\subsubsection{Analyse des critères partiels dans le système}

L'évolution des critères partiels (masse et pertes) des véhicules électriques le long du front optimal de Pareto dans le cas de la mission urbaine est donnée à la Figure 9. Les solutions trouvées sont à transmission directe sans réducteur de vitesse $\left(N=1\right.$ soit $P_{\text {red }}=0$ et $\left.M_{\text {red }}=0\right)$ et présentent toutes un onduleur de masse négligeable $\left(M_{\text {rad }} \approx 6 \mathrm{~kg}\right)$ par rapport aux autres masses dans le système.

L'étude des critères partiels associés aux solutions optimales permet d'identifier les éléments critiques du système. Dans le cas du véhicule électrique «pur », ce sont bien évidemment les caractéristiques de la batterie et du moteur de traction qui conditionnent le plus la valeur des critères de masse et de pertes.
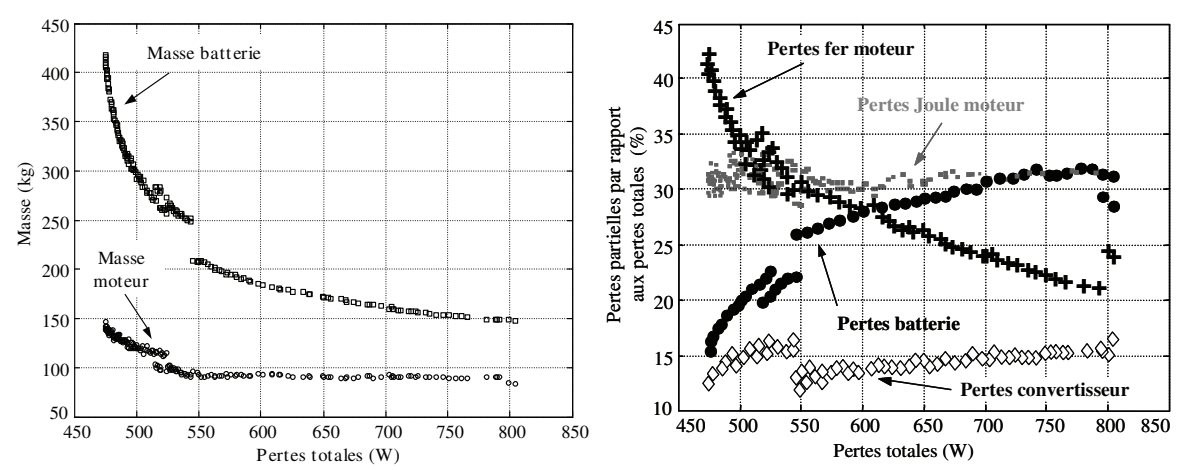

Figure 9. Critères partiels de masse et de pertes associés aux solutions Paretooptimales de véhicules électriques conçus pour une mission urbaine.

\subsubsection{Analyse des couplages dans le système}

De même qu'il est possible d'examiner l'influence des critères partiels en regardant leur évolution le long du front optimal, nous pouvons aussi observer 
graphiquement les couplages entre les différentes grandeurs physiques (variables associées à l'architecture ou au dimensionnement, contraintes et critères de conception) au sein du système. Nous illustrons, à la Figure 10, l'évolution de grandeurs fortement couplées le long du front de Pareto, à savoir le nombre d'éléments de la batterie, le nombre de conducteurs par encoche, la résistance statorique de la machine et le courant maximal dans la machine.

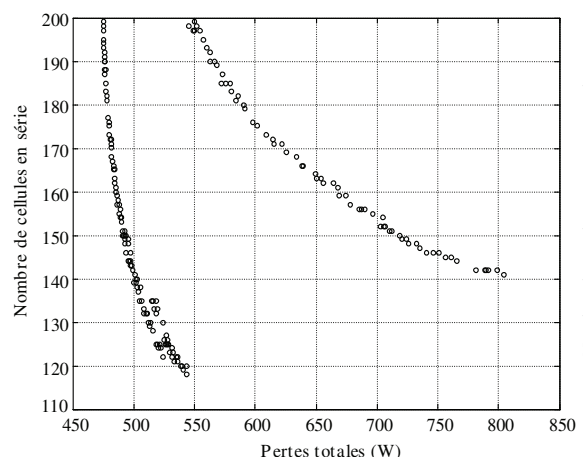

(a) Nombre de cellules en série de la batterie

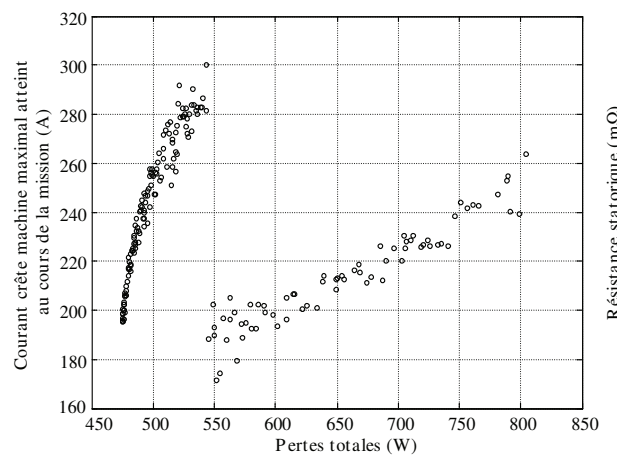

(c) Courant maximal dans la machine au cours de la mission

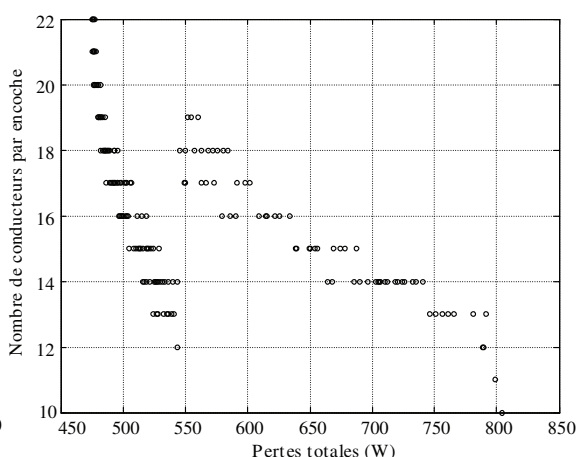

(b) Nombre de conducteurs par encoche dans la machine

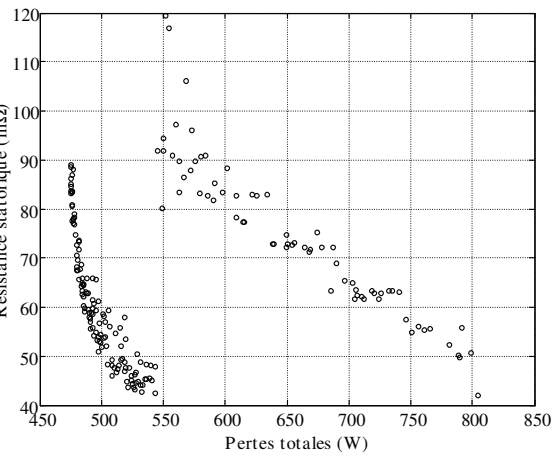

(d) Résistance statorique de la machine

Figure 10. Exemples de grandeurs couplées au sein du véhicule électrique

Dans certains, cas, il n'est pas toujours évident pour des variables continues de «visualiser» les couplages à partir de leur évolution le long du front de Pareto. Nous avons proposé une méthode d'analyse plus quantitative basée sur le calcul des coefficients de corrélation relatifs aux variations des variables de conception. Nous ne développerons pas ce point mais le lecteur pourra trouver de plus amples informations dans (Régnier, 2003), (Régnier et al., 2004). 


\subsubsection{Sensibilité des solutions optimales}

Nous avons déjà illustré la sensibilité des solutions optimales, notamment à travers l'influence de la mission de circulation. Il est aussi possible d'examiner leur robustesse vis-à-vis de variations d'autres paramètres (variables de conception, niveau d'une contrainte,...). En particulier, l'impact de modifications technologiques sur les critères de conception peut être quantifié. Cela peut permettre à l'expert d'identifier les parties «critiques » du système à améliorer en priorité pour atteindre de meilleures performances.

\section{Conclusions}

Dans cet article, nous avons abordé la notion de conception simultanée des systèmes énergétiques complexes et décrit les particularités des problèmes d'optimisation résultant de cette approche. La résolution de tels problèmes nécessite d'avoir recours à des méthodes multicritères, à variables mixtes, capables par ailleurs d'intégrer les diverses contraintes de conception. Les algorithmes évolutionnaires basés sur des méthodologies de type Pareto semblent aujourd'hui en bonne adéquation avec cette problématique. Ils assurent, d'une part, l'amélioration des performances des configurations à optimiser grâce à la détermination de l'ensemble des compromis optimaux. Ils permettent, d'autre part, à partir de la connaissance de cet ensemble, de déduire a posteriori des informations utiles associées aux systèmes comme l'influence des critères partiels, les couplages entre variables de conception, ou la sensibilité de paramètres vis-à-vis des performances : la richesse de telles informations aide le «concepteur système » dans sa démarche. Nous avons illustré ces différents aspects à travers un exemple concret de conception simultanée de système énergétique, à savoir la synthèse et le dimensionnement d'une chaîne de traction pour véhicules électriques destinés à remplir des cycles de fonctionnement urbain ou routier. Les résultats obtenus dans le cas d'un problème relativement complexe, à treize variables de conception et douze contraintes, nous encouragent à envisager des cahiers des charges plus conséquents présentant notamment des variations d'architecture plus importantes.

\section{Bibliographie}

Collette Y., Siarry P., Optimisation Multiobjectif, Paris, Eyrolles, 2002.

Corne D.W., Knowles J.D., Oates M.J., « The Pareto Envelope-based Selection Algorithm for Multiobjective Optimization », In M. Schoenauer, K. Deb, G. Rudolph, X. Yao, E. Lutton, J.J. Merelo and H.P. Schwefel eds., Proceedings of the Parallel Problem Solving from Nature VI Conference, 2000, Springer, p. 839-848.

Deb K., Agrawal S., Pratab A., Meyarivan T., «A fast-elitist non-dominated sorting genetic algorithm for multiobjective optimization: NSGA-II", Proceeding of the Parallel Problem Solving from Nature VI Conference, 2000, p. 849-858. 
Fonseca C.M., Fleming P.J., «Genetic Algorithms for Multiobjective Optimization: Formulation, Discussion and Generalization », In Stephanie Forrest, editor, Proceedings of the Fifth International Conference on Genetic Algorithms, San Mateo, California, 1993, p. 416-423.

Gillespie T.D., Fundamentals of vehicle dynamics, Society of automotive engineers, 1992

Hajela P., Lin C.Y., « Genetic search strategies in multicriterion optimal design », Structural Optimization, Vol. 4, 1992, p. 99-107.

Hoang E., Étude, modélisation et mesure des pertes magnétiques dans les moteurs à reluctance variable à double saillance, Thèse de l'Ecole Normale Supérieure de Cachan, 1995.

Horn J., Nafploitis N., Goldberg D.E., « A niched Pareto genetic algorithm for multiobjective optimization », Proceedings of the 1st IEEE Conference on Evolutionary Computation, New Jersey, 1994, p. 82-87.

Koza L., Genetic Programming: On the Programming of Computers by the means of natural selection, The MIT Press, Cambridge, Massachussets, 1992.

Kursawe F., "Evolution strategies for vector optimization », In Preliminary Proceedings of the Tenth International Conference on Multiple Criteria Decision Making, National Chiao Tung University, Taipei, China, 1992, p. 187-193.

Le Borzec R., «Réducteur de vitesse à engrenages », Techniques de l'Ingénieur, B5 640, 1999.

Michalewicz Z., Genetic Algorithms + Data Structures = Evolution Programs, 3ème edition, Springer-Verlag, Berlin Heidelberg New York, 1996

Multon B., «Motorisation des véhicules électriques », Techniques de l’ingénieur, E3 996, 2001, p 1-28.

Noble J., «Finding robust Texas Hold'em poker strategies using Pareto coevolution and deterministic crowding », Proceedings of the 1st International Conference on Machine Learning and Applications (ICLMA'02), CSREA Press, 2002.

Pareto V., Cours d'Economie Politique, Rouge, Lausanne, Suisse, 1896.

Rao S.S., Engineering Optimization - Theory and Practice, $3^{\text {ème }}$ edition, New York, John Wiley \& Sons, 1996.

Régnier J., Conception de systèmes hétérogènes en Génie Electrique par optimisation évolutionnaire multicritère, Thèse de doctorat, $n^{\circ} 2006$, Institut National Polytechnique de Toulouse, 2003.

Régnier J., Sareni B., Roboam X., «Optimal design of electrical engineering systems using Pareto Genetic Algorithms », 10th European Conference on Power Electronics and Applications, Toulouse, France, 2003

Régnier J., Sareni B., Roboam X, « System optimization by multiobjective genetic algorithms and analysis of the coupling between variables, constraints and objectives ${ }^{\circ} \gg$, Selected paper from the 8th International Workshop on Optimization and Inverse Problems in Electromagnetism (OIPE’04), COMPEL Journal, Vol. 24, №3, pp. 805-820, 2005 
Sareni B., Régnier J., Roboam X., «Recombination and Self-adaptation in Multi-objective Genetic Algorithms », Lecture Notes In Computer Science, Vol. 2936, P. Liardet et al Eds, 2004, Springer Verlag, p. 115-126.

Schaffer J.D., «Multiple objective optimization with vector evaluated genetic algorithms », Proceedings of the First International Conference on Genetic Algorithms and Their Applications, in J.J. Grefenstette ed., Pittsburgh, PA, 1985, p. 93-100.

Slemon G., Liu X., «Modeling and design optimization of permanent magnet motors », Electrical Machines and Power Systems, Vol. 20, 1992, p. 71-92.

Srinivas N., Deb K., «Multi-Objective function optimization using the non-dominated sorting genetic algorithm », Evolutionary Computation, Vol. 2, n³, 1994, p. 221-248.

Teich J., Blickle T., Thiele L., «An Evolutionary Approach to System-Level Synthesis », Proceedings of the 5th International Workshop on Hardware/Software Codesign, 1997, IEEE Computer Press Society, p. 167-172.

Trigui R., Badin F., Maillard P. Mailfert A, Étude de l'usage réel d'un véhicule utilitaire électrique, Revue Transport et Sécurité, N`50, Mars 1996, p.17-32.

Van Veldhuizen, D.A., Multiobjective Evolutionary Computation: Classifications, Analyses, and New Innovation, PhD Thesis, AFTI/DS/ENG/99-01, 1999.

Weile D.S, Michielssen E., Goldberg D.E., «Genetic Algorithm Design of Pareto Optimal Broadband Microwave Absorbers", IEEE Transactions on Electromagnetic Compatibility, Vol. 38, n³, 1996, p. 518-525.

Zitzler E., Evolutionary Algorithms for Multiobjective Optimization: Methods and Applications, PhD thesis, Swiss Federal Institute of Technology (ETH), Zurich, Suisse, 1999.

Zitzler E., Laumanns M., Thiele L., «SPEA2: Improving the Strength Pareto Evolutionary Algorithm », In K. Giannakoglou, D. Tsahalis, J. Periaux, P. Papailou and T. Fogarty (eds.) EUROGEN 2001, Evolutionary Methods for Design, Optimization and Control with Applications to Industrial Problems, Athènes, Grèce, 2001, p. 12-21. 


\section{Biographie}

Bruno Sareni est Maître de Conférences à l'Institut National Polytechnique de Toulouse, au département Génie Electrique et Automatique de l'ENSEEIHT et chercheur au Laboratoire d'Electrotechnique et d'Electronique Industrielle (LEEI). Ses activités de recherche au sein $d u$ groupe G-EnESys (groupe de recherche en énergie électrique et systémique) concernent la conception par optimisation des systèmes d'énergie électrique. Cette thématique l'amène à s'intéresser tout particulièrement à l'exploitation des méthodes évolutionnaires et des techniques d'optimisation liées à l'évolution artificielle.

Jérémi Régnier est Maître de Conférences à l'Institut National Polytechnique de Toulouse, au département Génie Electrique et Automatique de l'ENSEEIHT et chercheur au Laboratoire d'Electrotechnique et d'Electronique Industirelle. Sa thèse de doctorat, obtenu en 2003, traitait de la conception optimale de véhicules électriques à l'aide d'algorithmes évolutionnaires multicritères. Jérémi Régnier est aujourd'hui chercheur dans le Groupe CODIASE (commande et diagnostique des systèmes électriques) du LEEI et ses activités de recherche concernent notamment la surveillance et le diagnostique des systèmes électriques.

Xavier Roboam est Directeur de Recherche au CNRS et chercheur au LEEI. Il dirige actuellement le groupe de recherche en énergie électrique et systémique. Ses activités de recherche concernent notamment la conception des systèmes d'énergie électrique autonomes et des réseaux de bord embarqués. 